\title{
Numerical null controllability of a semi-linear heat equation via a least squares method
}

\author{
Enrique Fernández-Cara ${ }^{\mathrm{a}}$, Arnaud Münch ${ }^{\mathrm{b}}$ \\ ${ }^{a}$ Dpto. EDAN, University of Sevilla, Aptdo. 1160, 41080 Sevilla. \\ b Laboratoire de Mathématiques, Université Blaise Pascal (Clermont-Ferrand 2), UMR CNRS 6620, Campus des \\ Cézeaux, 63177 Aubière, France \\ Received $* * * * * ;$ accepted after revision +++++ \\ Presented by
}

\begin{abstract}
This note deals with the computation of distributed null controls for a semi-linear 1D heat equation, in the sublinear and slightly superlinear cases. Under sharp growth assumptions, the existence of controls has been obtained in [Fernández-Cara $\& 3$ Zuazua, Null and approximate controllability for weakly blowing up semi-linear heat equation, 2000] via a fixed point reformulation; see also [Barbu, Exact controllability of the superlinear heat equation, 2000]. More precisely, Carleman estimates and Kakutani's theorem together ensure the existence of fixed points for a corresponding linearized control mapping. In practice, the difficulty is to extract from the Picard iterates a convergent (sub)sequence. We introduce and analyze a least squares reformulation of the problem; we show that this strategy leads to an effective and constructive way to compute fixed points. To cite this article: E. Fernández-Cara, A. Münch, C. R. Acad. Sci. Paris, Ser. I 340 (2005).
\end{abstract}

\section{Résumé}

Contrôlabilité exacte à zéro d'une equation de la chaleur semi-linéaire par une méthode des moindres carrés. Cette note concerne la détermination effective de contrôles à zéro pour une équation de la chaleur semi-linéaire, dans le cas légèrement surlinéaire. Sous des conditions de croissances optimales, l'existence de contrôles a été obtenue dans [Fernández-Cara $\&$ Zuazua, Null and approximate controllability for weakly blowing up semi-linear heat equation, 2000] par un argument de point fixe; voir aussi [Barbu, Exact controllability of the superlinear heat equation, 2000]. Précisément, des inégalités de Carleman et le théorème de Kakutani impliquent l'existence de points fixes pour un opérateur de contrôle linéarisé associé. En pratique, la difficulté est d'extraire des itérés de Picard une sous-suite convergente. Cette note propose et analyse une reformulation du problème par une approche de type moindres carrés : on montre que celle-ci garantit une construction explicite de points fixes. Pour citer cet article : E. Fernández-Cara, A. Münch, C. R. Acad. Sci. Paris, Ser. I 340 (2005).

Email addresses: cara@us.es (Enrique Fernández-Cara), arnaud.munch@math.univ-bpclermont.fr (Arnaud Münch). 


\section{Version française abrégée}

Cette note concerne la nulle contrôlabilité de l'équation de la chaleur semi-linéaire. Plus précisement, on considère le système $(2)$, où $\omega \subset(0,1)$ désigne un ouvert non vide de $(0,1), T>0, a \in C^{1}([0,1])$ avec $a(x) \geq a_{0}>0, y_{0} \in L^{2}(0,1)$; la fonction $v \in L^{\infty}(\omega \times(0, T))$ est le contrôle et $y$ est l' état associé. On suppose que $f: \mathbb{R} \rightarrow \mathbb{R}$ est localement Lipschitzienne, $f(0)=0$ et satisfait (3), de sorte que (2) possède exactement une solution locale en temps. On rappelle également que sous la condition (4) de croissance sur $f$, les solutions de (2) sont globalement définies sur $[0, T]$ avec la régularité usuelle $y \in \mathcal{H}=C^{0}\left([0, T] ; L^{2}(0,1)\right) \cap L^{2}\left(0, T ; H_{0}^{1}(0,1)\right) ;$ voir [2].

Le système (2) est dit exactement contrôlable à zéro au temps $T$ si, pour toute donnée $y_{0} \in L^{2}(0,1)$, il existe un contrôle $v \in L^{\infty}\left(q_{T}\right)$ et un état associé $y$ défini globalement, tel que $y \in \mathcal{H}$ et $(5)$ soit vérifié. La référence [7] montre que, si la fonction $f$ vérifie (3) et ne croit pas à l'infini plus vite que $|s| \log ^{3 / 2}(1+|s|)$, alors (2) est contrôlable à zéro ; voir Théorème 1.1 et également [1] pour un résultat similaire.

Ce résultat est obtenu par un argument de point fixe, après linéarisation de (2) : précisément, on considère l'opérateur $\Lambda_{0}: L^{2}\left(Q_{T}\right) \rightarrow L^{2}\left(Q_{T}\right)$ qui à $z$ associe la solution $y_{z}$ controlée du système linéarisé (8), où la non linéarité $f(y)$ est remplacée par le terme $y g(z)$ avec $g$ défini par (6). Suivant [8], la solution $y_{z}$ et son contrôle $v_{z}$ sont choisis de façon à minimiser la fonctionnelle (9), faisant intervenir une norme $L^{2}$ à poids du contrôle et de la solution contrôlée. Les poids $\rho$ et $\rho_{0}$ sont définis par (7).

Dans un contexte similaire, [7] montre l'existence d'une constante $M>0$ telle que l'image de la boule fermée $B(0, M)$ de $L^{2}\left(Q_{T}\right)$ par $\Lambda_{0}$ reste dans $B(0, M)$. Le théorème de Kakutani assure alors l'existence d'au moins un point fixe pour $\Lambda_{0}$, mais ne garantit pas la convergence de la suite $\left\{y^{n}\right\}_{n>0}$ naturellement définie à partir de $\Lambda_{0}$ par $y^{n+1}=\Lambda_{0}\left(y^{n}\right), n \geq 0, y^{0}$ donné dans $L^{2}\left(Q_{T}\right)$. Pour cette raison, nous considérons le problème de minimisation (12) dont la solution globale est un point fixe de $\Lambda_{0}$, ou de façon équivalente, une solution controlée de (2). Dans (12), $\Lambda$ désigne la restriction de $\Lambda_{0}$ à $Z:=\left\{z \in L_{l o c}^{1}\left(Q_{T}\right) ; \iint_{Q_{T}}(T-t)^{-1}|z|^{2} d x d t<+\infty\right\}$.

On montre tout d'abord que, si $g \in C_{b}^{1}(\mathbb{R})$, alors la fonction $R$ de (12) est de classe $C^{1}(Z)$ et sa dérivée première est donnée par (13), voir Proposition 3.1. Ensuite, on montre que la norme $\left\|R^{\prime}(z)\right\|_{Z}$ est minorée, à une constante près $\mathcal{K}:=\left(1-K\left(\omega, T, a_{0},\|g\|_{L^{\infty}(\mathbb{R})}\right)\left\|g^{\prime}\right\|_{L^{\infty}(\mathbb{R})}\left\|y_{0}\right\|_{L^{\infty}}\right)$, par la norme $\| z-$ $\Lambda(z) \|_{Z}$; voir Proposition 3.2. Remarquablement, il en résulte que si la donnée initiale $y_{0}$ à contrôler est suffisamment petite de telle façon que $\mathcal{K}>0$, alors les points critiques de $R$ sont des points fixes de $\Lambda$, c'est-à-dire des solutions controlées à zéro pour le système non linéaire (2); voir Corollaire 3.1 .

Sous ces hypothèses, la résolution du problème (12) par une méthode de gradient génère la suite $\left\{z^{n}\right\}_{n \geq 0}$ définie par

$$
z^{n+1}=z^{n}-\rho R^{\prime}\left(z^{n}\right), \quad n \geq 0, \quad z^{0} \in Z
$$

convergeante vers une solution de (2). Nous renvoyons à [6] pour des expériences numériques mettant en évidence des données $\left(\omega, T, a_{0}, y_{0}\right)$ pour lesquelles la suite (1) converge tandis que la suite $\left\{y^{n}\right\}_{n \geq 0}$ des itérés de Picard, bien que restant borné dans $L^{2}\left(Q_{T}\right)$, ne converge pas. Mentionnons enfin que cette approche est générale et s'adapte à beaucoup d'autres systèmes contrôlables rentrant dans le cadre de [8].

\section{English version}

\section{Problem statement}

Let $\omega \subset(0,1)$ denote a (small) non-empty open interval, let $1_{\omega}$ be the associated characteristic function and let us assume that $T>0$ and $a \in C^{1}([0,1])$, with $a(x) \geq a_{0}>0, y_{0} \in L^{2}(0,1)$. We will consider the null controllability problem for the semi-linear 1-D heat system 


$$
\left\{\begin{array}{lr}
y_{t}-\left(a(x) y_{x}\right)_{x}+f(y)=v 1_{\omega}, & (x, t) \in Q_{T}=(0,1) \times(0, T) \\
y(x, t)=0, & (x, t) \in \Sigma_{T}=\{0,1\} \times(0, T) \\
y(x, 0)=y_{0}(x), & x \in(0,1) .
\end{array}\right.
$$

Here, $v \in L^{\infty}(\omega \times(0, T))$ is the control and $y$ is the associated state. We assume that $f: \mathbb{R} \rightarrow \mathbb{R}$ is, at least, locally Lipschitz-continuous, $f(0)=0$ and

$$
\left|f^{\prime}(s)\right| \leq C\left(1+|s|^{5}\right), \quad \text { p.p. } \quad s \in \mathbb{R},
$$

so that (2) possesses exactly one local in time solution. We recall (see [2]) that, under the growth condition

$$
|f(s)| \leq C(1+|s| \log (1+|s|)) \quad \forall s \in \mathbb{R},
$$

any solution is globally defined in $[0, T]$ and satisfies $y \in \mathcal{H}=C^{0}\left([0, T] ; L^{2}(0,1)\right) \cap L^{2}\left(0, T ; H_{0}^{1}(0,1)\right)$. Without such a condition, the solutions to (2) can blow up before $t=T$; the blow-up time depends on the sizes of $\left\|y_{0}\right\|_{L^{2}(0,1)}$ and $\|a\|_{L^{\infty}}$.

The system (2) is said to be null-controllable at time $T$ if, for any $y_{0} \in L^{2}(0,1)$, there exist controls $v \in L^{\infty}\left(q_{T}\right)$ and associated states $y$ that are again globally defined in $[0, T]$, belong to $\mathcal{H}$ and satisfy

$$
y(x, T)=0, \quad x \in(0,1) .
$$

Reference [7] provides conditions on $f$ under which (2) is null-controllable (see also [1]):

Theorem 1.1 ([7]) Let $T>0$ be given. Assume that $f: \mathbb{R} \rightarrow \mathbb{R}$ is locally Lipschitz-continuous, $f(0)=0$, (3) is satisfied and

$$
f(s)\left(|s| \log ^{3 / 2}(1+|s|)\right)^{-1} \rightarrow 0 \quad \text { as } \quad|s| \rightarrow \infty .
$$

Then (2) is null-controllable at time $T$.

Thus, if $f$ does not grow at infinity faster than $|s| \log ^{p}(1+|s|)$ for some $p<3 / 2$, the action of the control $v$ on $\omega \times(0, T)$ can compensate the blow up of the solution. The same result was obtained before in [4] for $p<1$. Let us also mention [3], where a positive positive boundary controllability result is obtained for a specific class of initial and final data and $T$ large enough.

\section{Linearization - A fixed point operator}

For simplicity, let us assume that $f \in C^{1}(\mathbb{R})$. Let us introduce the function $g$, with

$$
g(s)=s^{-1} f(s) \text { if } s \neq 0, g(0)=f^{\prime}(0) \text { otherwise. }
$$

Then $g \in C^{0}(\mathbb{R})$ and $f(s)=g(s) s$ for all $s$ (recall that $f(0)=0$ ). We will use the weights $\rho$ and $\rho_{0}$ introduced by Fursikov and Imanuvilov [8]:

$$
\left\{\begin{array}{l}
\rho(x, t)=\exp \left(\beta(x)(T-t)^{-1}\right), \rho_{0}(x, t)=(T-t)^{3 / 2} \rho(x, t), \beta(x)=K_{1}\left(e^{K_{2}}-e^{\beta_{0}(x)}\right) \\
\text { the } \left.K_{i} \text { are large positive constants (depending on } T, a_{0},\|a\|_{C^{1}} \text { and }\|A\|_{\infty}\right) \\
\text { and } \beta_{0} \in C^{\infty}([0,1]), \beta_{0}>0 \text { in }(0,1), \beta_{0}(0)=\beta_{0}(1)=0,\left|\beta_{0}^{\prime}\right|>0 \text { outside } \omega .
\end{array}\right.
$$

For any $z \in L^{1}\left(Q_{T}\right)$, we also introduce the notation

$$
L_{g(z)} y=y_{t}-\left(a(x) y_{x}\right)_{x}+g(z) y, \quad L_{g(z)}^{*} q=-q_{t}-\left(a(x) q_{x}\right)_{x}+g(z) q
$$

and we set $P_{0}=\left\{q \in C^{2}\left(\bar{Q}_{T}\right): q=0\right.$ on $\left.\Sigma_{T}\right\}$. In this linear space, the bilinear form

$$
m(z ; p, q):=\iint_{Q_{T}} \rho^{-2} L_{g(z)}^{*} p L_{g(z)}^{*} q d x d t+\iint_{q_{T}} \rho_{0}^{-2} p q d x d t
$$


is a scalar product, as a consequence of the unique continuation property. Let $P$ be the completion of $P_{0}$ for this scalar product. It can be shown that $P$ does not depend on $z$.

Let us consider the linear control systems

$$
L_{g(z)} y=v 1_{\omega}, \quad(x, t) \in Q_{T} ; \quad y(x, t)=0, \quad(x, t) \in \Sigma_{T} ; \quad y(x, 0)=y_{0}(x), \quad x \in(0,1) .
$$

Then, we define the mapping $\Lambda_{0}: L^{2}\left(Q_{T}\right) \mapsto L^{2}\left(Q_{T}\right)$ where, for any $z \in L^{2}\left(Q_{T}\right), y_{z}=\Lambda_{0}(z)$ is, together with $v_{z}$, the unique solution to the linear extremal problem

$$
\left\{\begin{array}{l}
\text { Minimize } J(z ; y, v):=\frac{1}{2} \iint_{Q_{T}} \rho^{2}|y|^{2} d x d t+\frac{1}{2} \iint_{q_{T}} \rho_{0}^{2}|v|^{2} d x d t \\
\text { Subject to } v \in L^{2}\left(q_{T}\right), \quad(y, v) \text { satisfies }(8) .
\end{array}\right.
$$

The couple $\left(y_{z}, v_{z}\right)$ is characterized as follows :

$$
y_{z}=\Lambda_{0}(z)=\rho^{-2} L_{g(z)}^{*} p_{z}, \quad v_{z}=-\left.\rho_{0}^{-2} p_{z}\right|_{q_{T}},
$$

where $p_{z}$ is the unique solution to the linear (elliptic) problem

$$
m\left(z ; p_{z}, q\right)=\int_{0}^{1} y_{0}(x) q(x, 0) d x \quad \forall q \in P ; \quad p_{z} \in P .
$$

The well-posedness of (10) is deduced from the estimate

$$
\|q(, 0)\|_{H_{0}^{1}(0,1)}^{2} \leq C_{0} m(z ; q, q) \quad \forall q \in P, \quad C_{0}=C_{0}\left(\omega, T, a_{0},\|a\|_{C^{1}},\|g\|_{L^{\infty}(\mathbb{R})}\right)>0,
$$

implying in particular that the right hand side of (10) is continuous with respect to the norm defined by $m$; see $[6,8]$. Therefore, in order to solve the null controllability problem for (2), it "suffices" to find a solution to the fixed point equation

$$
y=\Lambda_{0}(y), \quad y \in L^{2}\left(Q_{T}\right) .
$$

This fixed point formulation has been used in [7] to prove Theorem 1.1. Precisely, it is shown that there exists $M>0$ such that $\Lambda_{0}$ maps the closed ball $B(0 ; M) \subset L^{2}\left(Q_{T}\right)$ into itself. Then, Kakutani's theorem provides the existence of at least one fixed point for $\Lambda_{0}$. However, this property does not guarantee the convergence of the bounded sequence $\left\{y^{n}\right\}_{n \geq 0}$ naturally defined by $y^{n+1}=\Lambda_{0}\left(y^{n}\right)$ for all $n \geq 0$, with $y^{0}$ given in $L^{2}\left(Q_{T}\right)$.

\section{Least squares reformulation of the null controllability problem}

Let us introduce the function $\zeta(t) \equiv(T-t)^{-1 / 2}$ and the space $Z:=L^{2}\left(\zeta^{2}, Q_{T}\right)=\left\{z \in L_{l o c}^{1}\left(Q_{T}\right)\right.$ : $\left.\iint_{Q_{T}} \zeta^{2}|z|^{2} d x d t<+\infty\right\}$ and let us denote by $\Lambda$ the restriction to $Z$ of the mapping $\Lambda_{0}$. Obviously, $\Lambda(z) \in Z$ for all $z \in Z$.

Let us consider the following least squares reformulation of (11):

$$
\left\{\begin{array}{l}
\text { Minimize } R(z):=\frac{1}{2}\|z-\Lambda(z)\|_{Z}^{2} \\
\text { Subject to } z \in Z
\end{array}\right.
$$

Any solution to (11) solves (12). Conversely, if $y$ solves (12), we necessarily have $R(y)=0$ (because (2) is null controllable with control-states $(y, v)$ such that $J(z ; y, v)<+\infty)$; hence, $y$ also solves (11). Therefore (11) and (12) are, in the present context, equivalent.

The idea is to solve (12) by using gradient techniques. To this purpose, it is crucial to determine conditions under which $R$ is differentiable and, also, to compute $R^{\prime}(z)$. 
Proposition 3.1 Let us assume that $g \in C_{b}^{1}(\mathbb{R})$. Then $R \in C^{1}(Z)$. Moreover, for any $z \in Z$, the derivative $R^{\prime}(z)$ is given by

$$
R^{\prime}(z)=\left(1-\rho^{-2} g^{\prime}(z) p_{z}\right)\left(z-y_{z}\right)+\zeta^{-2} g^{\prime}(z)\left(y_{z} \lambda_{z}+p_{z} \mu_{z}\right),
$$

where $p_{z}$ is the unique solution to (10), $y_{z}=\rho^{-2} L_{g(z)}^{*} p_{z}, \lambda_{z}$ is the unique solution to the linear (adjoint) problem

$$
m\left(z ; q, \lambda_{z}\right)=\left(z-y_{z}, \rho^{-2} L_{g(z)}^{*} q\right)_{Z} \quad \forall q \in P ; \quad \lambda_{z} \in P,
$$

and, finally, $\mu_{z}=\rho^{-2} L_{g(z)}^{*} \lambda_{z}$.

The behavior of $R^{\prime}(z)$ is explained in the following result:

Proposition 3.2 Let the assumptions in Proposition 3.1 be satisfied. There exists a constant $K$ that depends on $\omega, T, a_{0},\|a\|_{C^{1}}$ and $\|g\|_{L^{\infty}(\mathbb{R})}$ but is independent of $z$ and $y_{0}$, such that the following holds for all $z \in Z$ :

$$
\left\|R^{\prime}(z)\right\|_{Z} \geq\left(1-K\left\|g^{\prime}\right\|_{L^{\infty}(\mathbb{R})}\left\|y_{0}\right\|_{L^{\infty}}\right)\|z-\Lambda(z)\|_{Z} .
$$

A very relevant consequence of Proposition 3.2 is the following:

Corollary 3.1 Let us the assumptions in Proposition 3.1 be satisfied and let $K$ be the constant furnished by Proposition 3.2. If

$$
K\left\|g^{\prime}\right\|_{L^{\infty}(\mathbb{R})}\left\|y_{0}\right\|_{L^{\infty}}<1,
$$

then the critical points of $R$ are global minima and, consequently, solve (11).

For the proof of Proposition 3.2, the following technical lemmas are needed:

Lemma 3.2 For any $q \in P$ one has $(\zeta \rho)^{-1} q \in L^{\infty}\left(Q_{T}\right)$. Furthermore, there exists $C>0$, only depending on $\omega, T, a_{0},\|a\|_{C^{1}}$ and $\|g\|_{L^{\infty}(\mathbb{R})}$, such that

$$
\left\|(\zeta \rho)^{-1} q\right\|_{L^{\infty}\left(Q_{T}\right)}^{2} \leq C\|q\|_{P}^{2} \quad \forall q \in P .
$$

Lemma 3.3 With the notation of Proposition 3.1, one has:

$$
\left\|p_{z}\right\|_{P} \leq C\left\|y_{0}\right\|_{L^{\infty}} \quad \forall z \in Z
$$

and

$$
\left\|\lambda_{z}\right\|_{P} \leq C\left\|\zeta \rho^{-1}\right\|_{\infty}\left\|z-y_{z}\right\|_{Z} \quad \forall z \in Z,
$$

where $C$ depends on $\omega, T, a_{0},\|a\|_{C^{1}}$ and $\|g\|_{L^{\infty}(\mathbb{R})}$.

We refer to [6] for the proofs of these lemmas, based on global Carleman inequalities.

Proof of Proposition 3.2 - Let $z \in Z$ be given and let us introduce $f:=z-y_{z}$. In view of Proposition 3.1, one has

$$
\begin{aligned}
& \left\|R^{\prime}(z)\right\|_{Z} \geq \frac{1}{\|f\|_{Z}}\left(R^{\prime}(z), f\right) \\
& \quad=\frac{1}{\|f\|_{Z}} \iint_{Q_{T}}\left(\zeta^{2}\left(1+\rho^{-2} g^{\prime}(z) p_{z}\right)|f|^{2}+g^{\prime}(z)\left(y_{z} \lambda_{z}+p_{z} \mu_{z}\right) f\right) d x d t \\
& \quad \geq\|f\|_{Z}-\frac{1}{\|f\|_{Z}} \iint_{Q_{T}} \zeta^{2} \rho^{-2}\left|g^{\prime}(z)\right|\left|p_{z}\right||f|^{2} d x d t-\frac{1}{\|f\|_{Z}} \iint_{Q_{T}}\left|g^{\prime}(z)\right|\left(\left|y_{z} \| \lambda_{z}\right|+\left|p_{z}\right|\left|\mu_{z}\right|\right)|f| d x d t .
\end{aligned}
$$

In view of Lemmas 3.2 and 3.3,

$$
\begin{aligned}
& \iint_{Q_{T}} \zeta^{2} \rho^{-2}\left|g^{\prime}(z)\right|\left|p_{z}\right||f|^{2} d x d t \leq\left\|\rho^{-2} g^{\prime}(z) p_{z}\right\|_{\infty}\left(\iint_{Q_{T}} \zeta^{2}|f|^{2} d x d t\right) \\
& \quad \leq\left\|\zeta \rho^{-1}\right\|_{\infty}\left\|g^{\prime}(z)\right\|_{\infty}\left\|(\zeta \rho)^{-1} p_{z}\right\|_{\infty}\|f\|_{Z}^{2} \leq C G_{1}\left\|p_{z}\right\|_{P}\|f\|_{Z}^{2} \leq C G_{1}\left\|y_{0}\right\|_{L^{\infty}}\|f\|_{Z}^{2},
\end{aligned}
$$


where $G_{1}:=\left\|g^{\prime}\right\|_{L^{\infty}(\mathbb{R})}$. On the other hand, from Lemma 3.2 we also have

$$
\begin{aligned}
& \iint_{Q_{T}}\left|g^{\prime}(z)\right|\left(\left|y_{z}\right|\left|\lambda_{z}\right|+\left|p_{z} \| \mu_{z}\right|\right)|f| d x d t \\
& \quad \leq\left\|g^{\prime}(z)\right\|_{\infty}\left[\left(\iint_{Q_{T}} \rho^{-2}\left|L_{g(z)}^{*} p_{z}\right|^{2} d x d t\right)^{1 / 2}\left\|(\zeta \rho)^{-1} \lambda_{z}\right\|_{\infty}\right. \\
&\left.+\left(\iint_{Q_{T}} \rho^{-2}\left|L_{g(z)}^{*} \lambda_{z}\right|^{2} d x d t\right)^{1 / 2}\left\|(\zeta \rho)^{-1} p_{z}\right\|_{\infty}\right]\left(\iint_{Q_{T}} \zeta^{2}|f|^{2} d x d t\right)^{1 / 2} \\
& \quad \leq C G_{1}\left[\left\|y_{0}\right\|_{L^{\infty}}\left\|\lambda_{z}\right\|_{P}+\left\|p_{z}\right\|_{P}\left\|\zeta \rho^{-1}\right\|_{\infty}\|f\|_{Z}\right]\|f\|_{Z} \\
& \leq C G_{1}\left\|y_{0}\right\|_{L^{\infty}}\|f\|_{Z}^{2} .
\end{aligned}
$$

Consequently, $\left\|R^{\prime}(z)\right\|_{Z} \geq\|f\|_{Z}-K\left\|g^{\prime}\right\|_{L^{\infty}(\mathbb{R})}\left\|y_{0}\right\|_{L^{\infty}}\|f\|_{Z}$ and we get (14) for some $K$.

It is therefore very appropriate to try to solve the null controllability problem for (2) by applying a gradient method to the extremal problem (12). Indeed, under the hypotheses of Corollary 3.1, the sequence $\left\{z^{n}\right\}_{n \geq 0}$ defined by (1) converges, as $n \rightarrow+\infty$, toward a fixed point of $\Lambda$. The limit $\tilde{z}$ and its associated control $v_{\tilde{z}}$ solve (2) and satisfy (5). We refer to [6] for details and for some numerical experiments with $f(s)=C s \log ^{p}(1+|s|), C>0, p \in \mathbb{R}$, exhibiting data for which the sequence $\left\{z^{n}\right\}_{n \geq 0}$ given by (1) converges, in contrast with the sequence $\left\{y^{n}\right\}_{n \geq 0}$ defined by $y^{n+1}=\Lambda\left(y^{n}\right), n \geq 1$.

Let us finally mention that this least squares approach can also be used in the context of many other controllable systems for which appropriate Carleman estimates are available: Navier-Stokes systems, wavelike equations, etc.; see [8], [9].

\section{References}

[1] V. Barbu, Exact controllability of the superlinear heat equation, Appl. Math. Optim. Optimization, Theory and Applications 42(1), (2000) 73-89.

[2] T. Cazenave, A. Haraux, Introduction aux problèmes d'évolutions semi-linéaires, Mathématiques et Applications, Ellipses, Paris 1989.

[3] J-M. Coron, E. Trélat, Global steady-state controllability of one dimensional semilinear heat equations, SIAM J. Control Optim. 43(2) 2004, 549-569.

[4] E. Fernandez-Cara, Null controllability of the semi-linear heat equation, Esaim:COCV (1997), no. 2, 87-103.

[5] E. Fernandez-Cara and A. Münch, Numerical null controllability of the 1-d heat equation: primal algorithm, Submitted to SIAM J. Control Optim.

[6] E. Fernandez-Cara and A. Münch, Numerical null controllability of a semi-linear 1-d heat equation, Preprint.

[7] E. Fernandez-Cara and E. Zuazua, Null and approximate controllability for weakly blowing up semilinear, Ann. Inst. Henri Poincaré, Analyse non linéaire. 17 (2000), no. 5, 583-616.

[8] A.V. Fursikov and O. Yu. Imanuvilov, Controllability of Evolution Equations, Lecture Notes Series, number 34. Seoul National University, Korea, (1996) 1-163.

[9] I. Lasiecka and R. Triggiani, Exact controllability of semilinear abstract systems with applications to waves and plates boundary control, Appl. Math. \& Optim., 23 (1991), 109-154. 